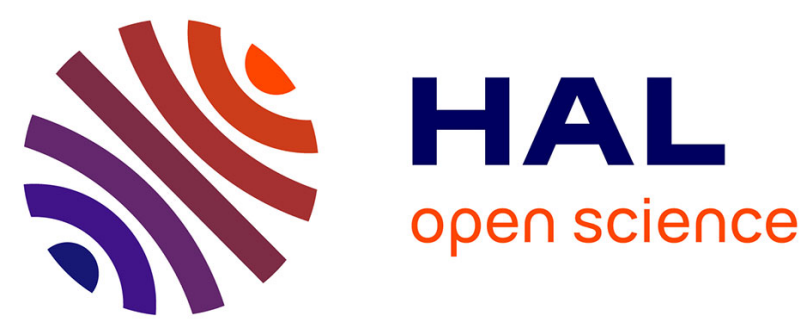

\title{
Quadrotors Data Fusion using a Particle Filter
}

\author{
Diego Mercado, Pedro Castillo, R Castro, Rogelio Lozano
}

\section{To cite this version:}

Diego Mercado, Pedro Castillo, R Castro, Rogelio Lozano. Quadrotors Data Fusion using a Particle Filter. International Conference on Unmanned Aircraft systems (ICUAS 2014), May 2014, Denver, United States. pp.890-894. hal-01137436

\section{HAL Id: hal-01137436 https://hal.science/hal-01137436}

Submitted on 30 Mar 2015

HAL is a multi-disciplinary open access archive for the deposit and dissemination of scientific research documents, whether they are published or not. The documents may come from teaching and research institutions in France or abroad, or from public or private research centers.
L'archive ouverte pluridisciplinaire HAL, est destinée au dépôt et à la diffusion de documents scientifiques de niveau recherche, publiés ou non, émanant des établissements d'enseignement et de recherche français ou étrangers, des laboratoires publics ou privés. 


\title{
Quadrotors Data Fusion using a Particle Filter*
}

\author{
D. A. Mercado ${ }^{1}$, P. Castillo ${ }^{1,2}$ and R. Lozano ${ }^{1,2}$
}

\begin{abstract}
This paper presents a data fusion algorithm by means of a particle filter (PF), for estimate the position of a quadrotor equipped with multiple sensors. A global positioning system (GPS) is considered for position measurement in a loosely coupled scheme, velocity can be obtained from an optic flow sensor, whilst an inertial measurement unit (IMU) can provide orientation and angular rate measurements. Simulations were carried out, where real noise from a not expensive GPS is added to the simulated position to test the proposed algorithm.
\end{abstract}

\section{INTRODUCTION}

Unmanned Aerial Vehicles (UAVs) have gained great relevance in the last years thanks to their huge potential in many civilian and military applications such as exploration, surveillance, search and rescue, in a cheap way and without risking human lives in dangerous situations. Particularly, four rotors rotorcrafts, also known as quadrotors, have received special interest since their rotor's configuration produces cancellation of the reactive torques, considerably simplifying their analysis and control. Also, they are suitable for vertical take off and landing, as well as hovering, making them a good choice for maneuvering in small spaces or perform high precision tasks.

Several stabilization and trajectory tracking control laws have been proposed for this kind of systems and validated through simulations and experiments in indoor applications, relying on a good measurement of the position and velocity. To cite some examples, [1] proposes an attitude stabilization control strategy for hover flight using nested saturations, while in [2] a sliding mode approach is used to accomplish position control of a quadrotor. In [3] a trajectory tracking control by means of a discrete time feedback linearization

\footnotetext{
*This work was supported by project SEARCH

${ }^{1}$ HEUDIASYC UMR 7253 CNRS-UTC, Compiègne, France. dmercado, pedro.castillo, rlozano@hds.utc.fr

${ }^{2}$ LAFMIA UMI 3175 CINVESTAV-CNRS, Mexico.
}

control scheme is proposed.

However, big effort is still required to accomplish autonomous flight for outdoor applications, due to presence of external perturbations, especially the wind, and the lack of a good measurement for the position and velocity. Not expensive Global Positioning Systems (GPS) sensors can provide an estimation of the position and velocity, however, the errors, of $2 \mathrm{~m}$ at best, and their low measurement rate of about $5 \mathrm{~Hz}$, are not suitable for precise applications and can interfere with the system stability, even more, GPS can easily lost its signal leaving the system without a position measurement. Another alternative widely studied are the optical flow sensors which use computer vision algorithms for estimating the motion velocity of a system, but they are noisy and sensibles for lighting changes. Data fusion algorithms are an interesting solution to this problem, they take information from multiple sensors, especially GPS, cameras and IMU, to improve the estimation of the position and velocity. The Kalman filter an its variations are the mos popular approach, for example, in [5] an observer-control scheme for quadrotors using the Extended Kalman Filter (EKF) is proposed and tested in real time indoor experiments, using data from an optic flow algorithm and an IMU. In [6], a Robust Adaptive Kalman Filter is proposed for estimation of UAV dynamics in the presence of sensor faults (see also [7], [8], [9]). However, this techniques assume that the process and measurement noise are Gaussian, which is not the case of a GPS in a loosely coupled scheme. The great advances in micro controllers processing capacity allow to explore other alternatives. The Particle Filter (PF) presents an interesting option to this problems, at the cost of computational expense, because it is not restricted to Gaussian noise. In ?? is presented a vision based algorithm using a particle filter tracking for estimating the position of a quadrotor. In this paper the use of the 


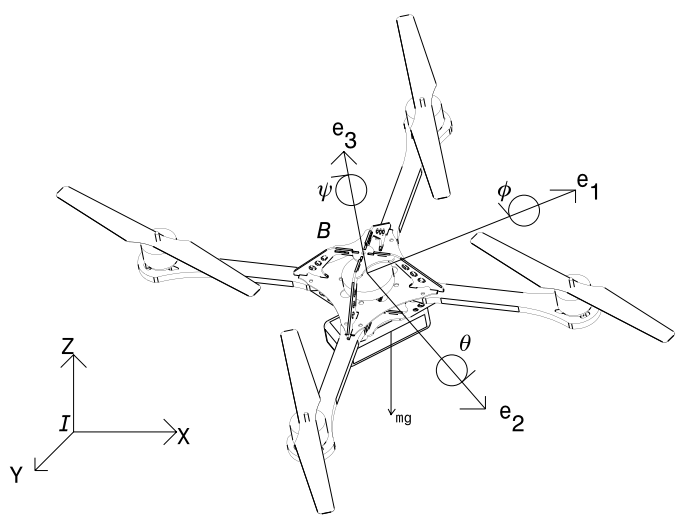

Fig. 1. Quadrotor in an inertial reference frame.

PF is explored, through simulations, for data fusion and state estimation in quadrotors equipped with multiple sensors in outdoor missions. Particularly, a GPS is considered for position measurement. Real GPS data was collected and the obtained error was added to the simulated position.

The paper proceeds as follows. In section II the dynamic model of the quadrotor is given. In section III, the PF for the quadrotor state estimation is proposed. In section IV the performance of the $\mathrm{PF}$ is tested in simulation. Section $\mathrm{V}$ gives the conclusions and future work.

\section{QUADROTOR DYNAMIC MODEL}

The quadrotor can be represented as a rigid body in space with mass $m$ and inertia matrix $J$, subject to gravitational and aerodynamic forces. Let us consider an inertial coordinate frame $I=\{X Y Z\}$, fixed to the ground and a body fixed coordinate frame, $B=\left\{e_{1}, e_{2}, e_{3}\right\}$ (see Fig. 1). Consider the vectors

$$
\begin{gathered}
\xi=\left[\begin{array}{lll}
x & y & z
\end{array}\right]^{T} \\
\Phi=\left[\begin{array}{lll}
\phi & \theta & \psi
\end{array}\right]^{T}
\end{gathered}
$$

which stand for the position of the center of gravity, with respect to the inertial frame $I$, and the Euler angles roll, pitch and yaw, respectively. The motion equations are given by the NewtonEuler equations in the inertial frame $I$ [10]

$$
\begin{aligned}
m \ddot{\xi} & =\mathrm{TRe}_{3}-m g e_{3} \\
J \dot{\Omega} & =-\Omega_{x} J \Omega+\Gamma
\end{aligned}
$$

where $T \in \mathfrak{R}^{+}$is the total thrust of the motors, $g$ is the gravity constant and $\Gamma \in \mathfrak{R}^{3}$ is the input torque defined in the body fixed frame $B . R \in S O(3): B \rightarrow$ $I$ is the rotational matrix from the body frame to the inertial frame.

$$
\Omega=\left[\begin{array}{lll}
p & q & r
\end{array}\right]^{T}
$$

represents the angular velocity in the body frame $B . \Omega_{x}$ stands for the skew symmetric matrix such that $\Omega_{x} v=\Omega \times v$ is the vector cross product. The kinematic relation between the generalized velocities $\dot{\Phi}=(\dot{\phi}, \dot{\theta}, \dot{\psi})$ and the angular velocity $\Omega$ is given by [12]

$$
\Omega=Q \dot{\Phi}
$$

with

$$
Q=\left[\begin{array}{ccc}
1 & 0 & -s \theta \\
0 & c \phi & c \theta s \phi \\
0 & -s \phi & c \theta c \phi
\end{array}\right]
$$

III. PARTICLE FILTER

Consider the state vector $\chi \in \mathfrak{R}^{12}$

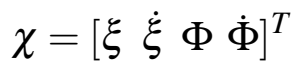

Then from (3), (4), with a sampling time $T_{s}$ small enough, the discrete time evolution model is given by

$$
\dot{\chi}_{k}=\left[\begin{array}{c}
\dot{\xi}_{k-1} T_{s}+\xi_{k-1} \\
\left(\frac{1}{m} T_{k-1} R_{k-1} e_{3}-g e_{3}\right) T_{s}+\dot{\xi}_{k-1} \\
\dot{\Phi}_{k-1} T_{s}+\Phi_{k-1} \\
\bar{\Gamma}_{k-1} T_{s}+\dot{\Phi}_{k-1}
\end{array}\right]+\omega_{k}
$$

where the total thrust $T$ and the control torque $\bar{\Gamma}$ are the system inputs. $\omega$ stands for the model noise with a probability density $p_{\omega}$.

Consider also the measurement vector $Y=$

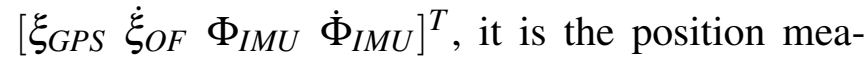
sured by a GPS, the velocity measured by an optic flow sensor and the orientation and angular rate obtained by an IMU. Hence, the observation model is

$$
Y_{k}=\chi_{k}+v_{k}
$$

$v$ represents the measurement noise with a probability density $p_{v}$.

The particle filter (PF) or sequential Monte Carlo technique is a kind of recursive Bayesian filter for state estimation [13]. It consists in generating $N_{p}$ 
particles according to the a priori probability, with an importance weight $W$ defined as

$$
W_{k}=\frac{p\left(Y_{1: k} \mid \chi_{0: k}\right) p\left(\chi_{0: k}\right)}{q\left(\chi_{0: k} \mid Y_{1: k}\right)}
$$

where $p(Y \mid \chi)$ is the probability density function of $Y$ given $\chi$, and $q\left(\chi_{k} \mid Y_{k}\right)$ is the so-called proposal distribution.

At each iteration, the outputs are measured by the sensors and the weights can be updated recursively, using the bootstrap method, by the following expression

$$
\begin{gathered}
W_{k}^{i}=W_{k-1}^{i} p\left(Y_{k} \mid \chi_{k}^{i}\right)=W_{k-1}^{i} p_{v}\left(Y_{k}-\chi_{k}^{i}\right) \\
i=1, \ldots, N_{p}
\end{gathered}
$$

for a normal probability distribution in the measurement, with covariance matrix $Q$

$$
W_{k}^{i}=W_{k-1}^{i} e^{\left(-\frac{1}{2}\left(Y_{k}-\chi_{k}^{i}\right) Q^{-1}\left(Y_{k}-\chi_{k}^{i}\right)\right)}
$$

normalizing

$$
\bar{W}_{k}^{i}=\frac{W_{k}^{i}}{\sum_{i=1}^{N_{p}} W_{k}^{i}}
$$

Then, the set of particles evolves following the evolution model (9), with $\omega_{k}^{i}$ randomly generated according to $p_{\omega}$.

In order to avoid the weight degeneracy problem, a resampling step is required. In order to do so, the effective sample size $N_{\text {eff }}$ is employed as a measure of the number of active particles

$$
N_{\text {eff }}=\frac{1}{\sum_{i=1}^{N_{p}}\left(W_{k}^{i}\right)^{2}}
$$

it can be noticed that this value is maximum $\left(N_{e f f}=N_{p}\right)$ when all the particles have the same weight, and minimum $\left(N_{e f f}=1\right)$ when only one weight is different from zero. If the effective sample size is lower than a certain threshold $N_{e f f}<$ $N_{t h}$, the resampling step is performed by means of the Kitagawa method [14].

The estimated stated is given by the particles barycenter, it is

$$
\bar{\chi}_{k}=\Sigma_{i=1}^{N_{p}} W_{k}^{i} \chi_{k}^{i}
$$

Since GPS sample frequency is slow $(5 \mathrm{~Hz})$ with respect to the others sensors, and in order to deal with the GPS loss signal, the measured position is updated in the following form

$$
\xi_{G P S k}=\xi_{G P S k-n}+n T_{s} \dot{\xi}_{k}
$$

where $n$ is the number of sample times $T_{S}$ passed since the last valid GPS data.

\section{SIMULATIONS}

Simulations were carried out to test the proposed estimation algorithm for a quadrotor with an inaccurate position measurement, which is the case for a not expensive GPS. GPS errors were obtained from real experiments in an urban environment surrounded by buildings, and added to the simulated actual position, taking also into account the GPS measurement rate $(5 \mathrm{~Hz})$ and including signal losses of 20 seconds every 100 seconds. The described GPS errors can be depicted in Fig. 2, where the zero values every 100 seconds represent signal losses. Optical flow sensors can provide a noisy velocity measurement, hence some with noise was added to the velocity signal. The noisy measured velocity is shown in Fig. 3 .

The performance of the particle filter for position estimation while the UAV evolves in a spiral trajectory can be seen in Fig. 4, using $N_{p}=100$ particles and a resampling threshold of $N_{t h}=10$. It can be noticed that the PF performs an excellent position estimation despite the poor position measurement, this is clearer from Fig. 5 where the estimation error is displayed, which can be compared against the measurement error in Fig. 2. It is clear that the position estimation greatly improved the measured one and perfectly handled the signal losses and from measurement errors of until 20 meters are obtained error of less than one meter, making it suitable for precision tasks. In order to provide a comparison against other estimation techniques, the popular EKF was also tested under the same conditions and the obtained estimation errors are presented at Fig. 6, where it can be seen that the errors are much higher than the obtained with the PF, this can be explained due to the fact that the EKF assumes that measurement noise is Gaussian, which is not the case for a GPS in a loosely coupled scheme (see Fig. 2). Finally, Figs. 7 and 8 contain the XY plane view and the three dimensional one with the real and estimated positions. 


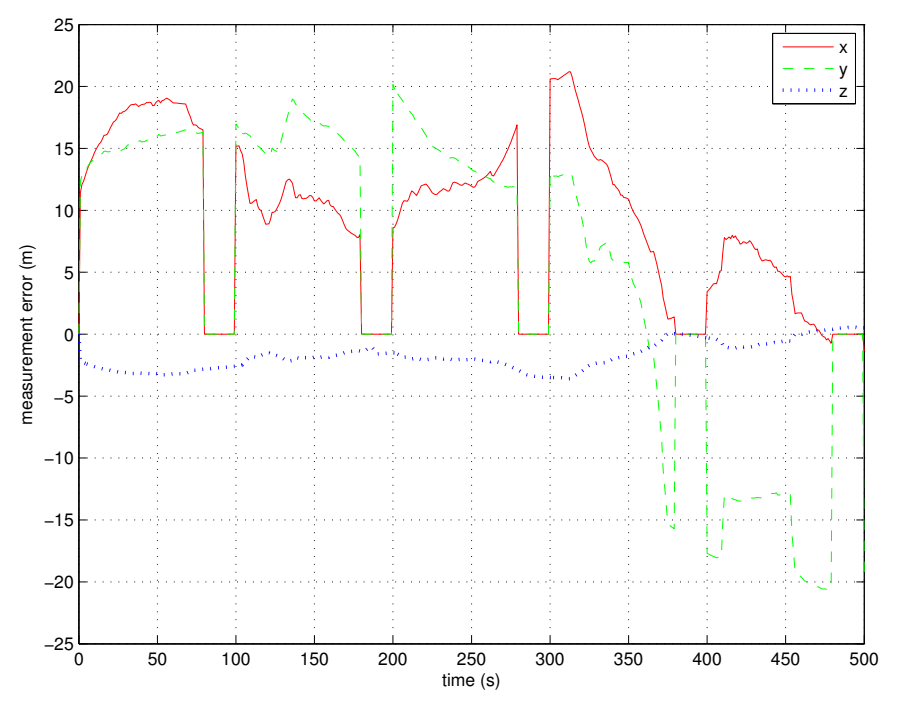

Fig. 2. GPS measurement error.

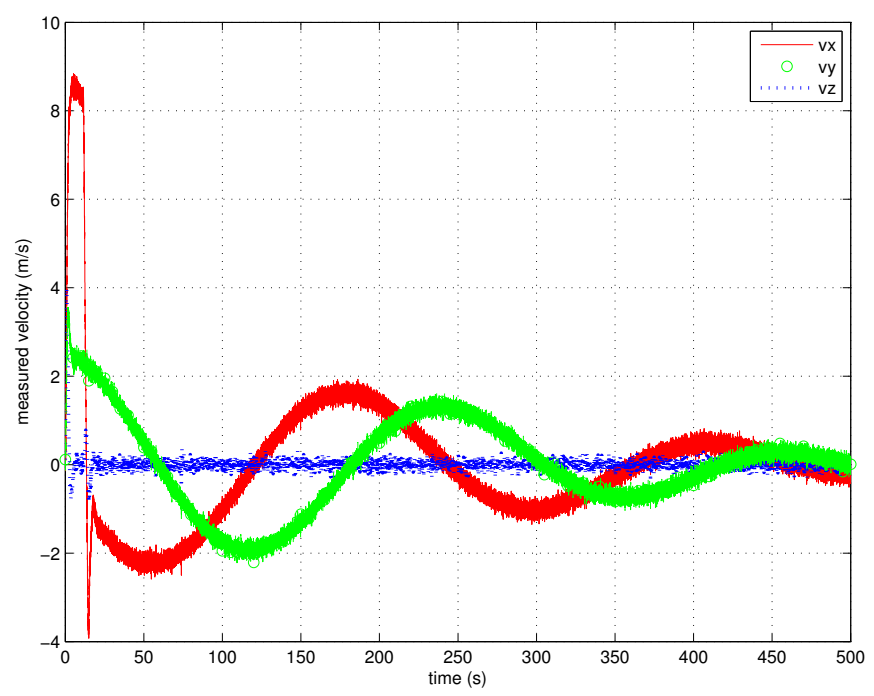

Fig. 3. Measured velocity.

\section{CONCLUSIONS AND FUTURE WORK}

Simulations have shown promising results of the proposed data fusion strategy, considerably reducing the measurement error while handling the GPS signal losses.

The PF proved to be more adequate in this case than the EKF, since the measurement noise of a GPS in a loosely coupled scheme is not Gaussian. Future work includes to implement the proposed strategy embedded on a quadrotor equipped with a GPS sensor, for real time experiments.

It is also desired to use the estimated data to
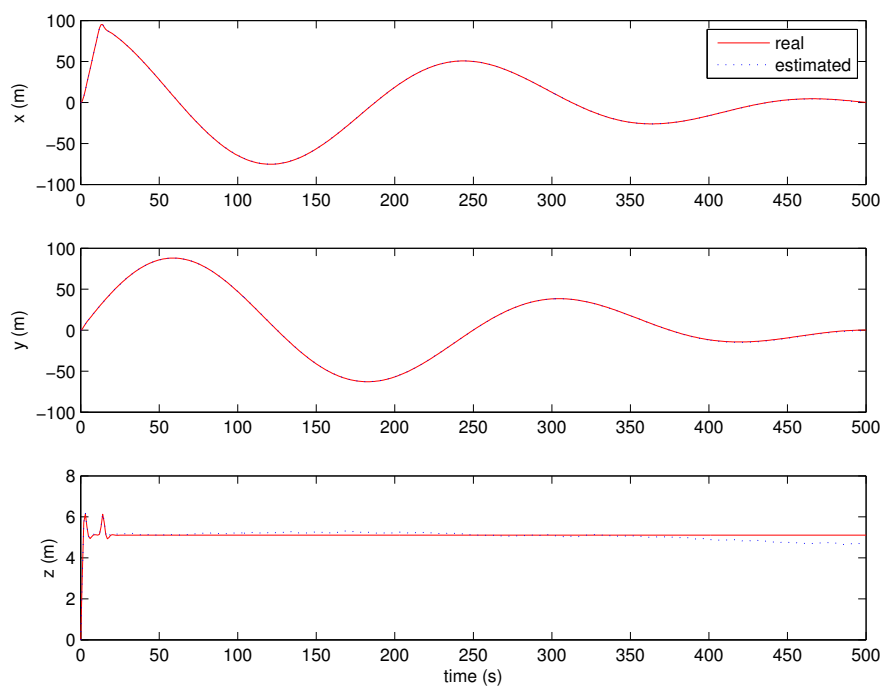

Fig. 4. Position.

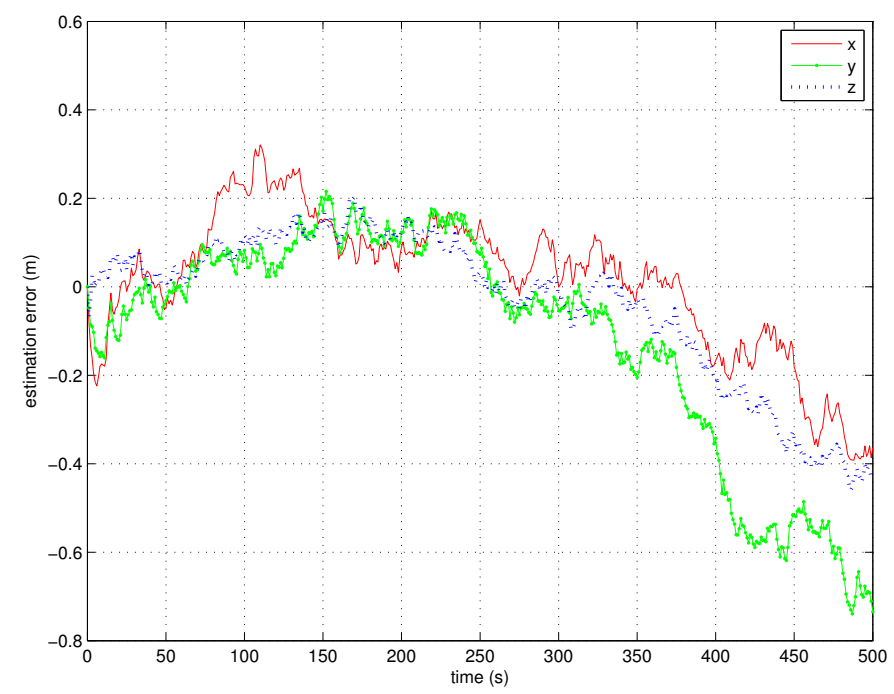

Fig. 5. PF estimation error.

close the loop in a control strategy to accomplish trajectory tracking for outdoor applications.

\section{REFERENCES}

[1] P. Castillo, R. Lozano, \& A. Dzul. Modelling and control of mini-flying machines. Springer-Verlag, London, 2005.

[2] R. Xu \& Ü. Özgüner. Sliding mode control of a quadrotor helicopter. En Proc. 45th IEEE Conference on Decision \& Control, 2006.

[3] M. Guisser, H. Medromi, J. Saadi \& H. Ifassiouen. Stabilization and trajectory tracking in discrete-time of an autonomous four rotor mini-rotorcraft. In Proceedings of the 2th International Symposium on Communications, Control and Signal Processing, 2006. 


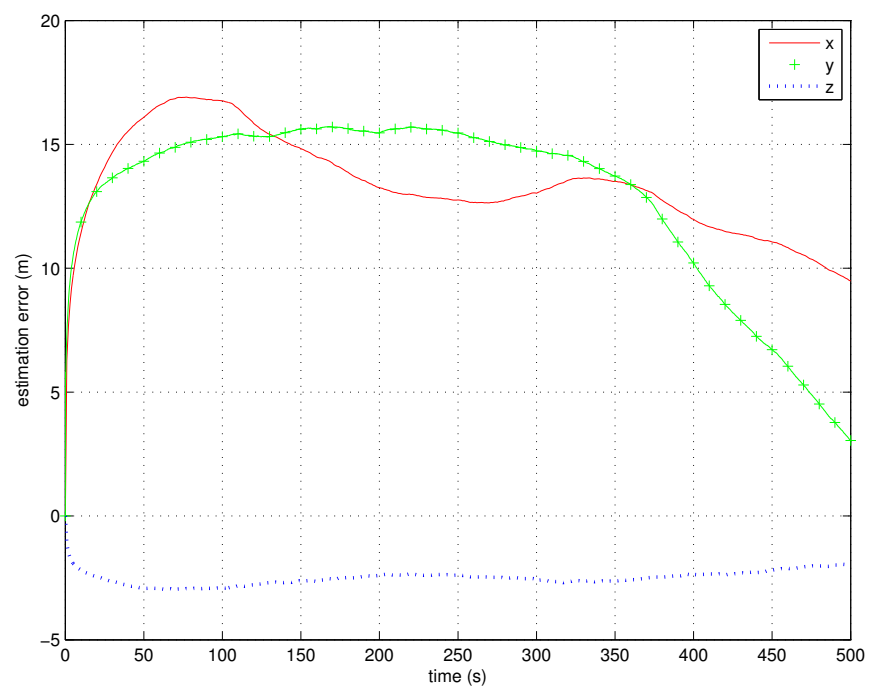

Fig. 6. EKF estimation error.

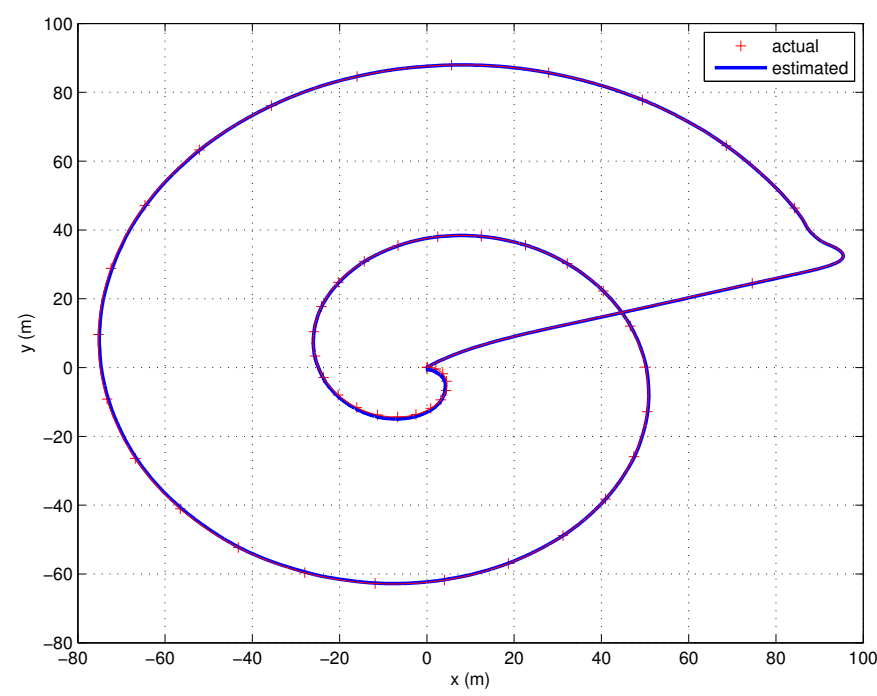

Fig. 7. XY plane.

[4] L. Derafa, L. Fridman, A. Benallegue \& Ouldali. Super Twisting Control Algorithm for the Four Rotors Helicopter Attitude Tracking Problem. In 11th International Workshop on Variable Structure Systems, Mexico City, Mexico, 2010.

[5] L. E. Munoz, P. Castillo \& P. Garcia. Observer-control scheme for autonomous navigation: Flight tests validation in a quadrotor vehicle. In International Conference on Unmanned Aircraft Systems (ICUAS), Atlanta, 2013.

[6] Hajiyev, C. \& Ersin, H. Robust Adaptive Kalman Filter for estimation of UAV dynamics in the presence of sensor/actuator faults. Aerospace Science and Technology 28, 376-383, 2013.

[7] M. George \& S. Sukkarieh. Tightly coupled INS/GPS with bias estimation for UAV applications. In Proceedings of the 2005 Australian Conferences on Robotics and Automation., 2005.

[8] W. Ding, J. Wang \& A. Almagbile. Adaptive filter design for

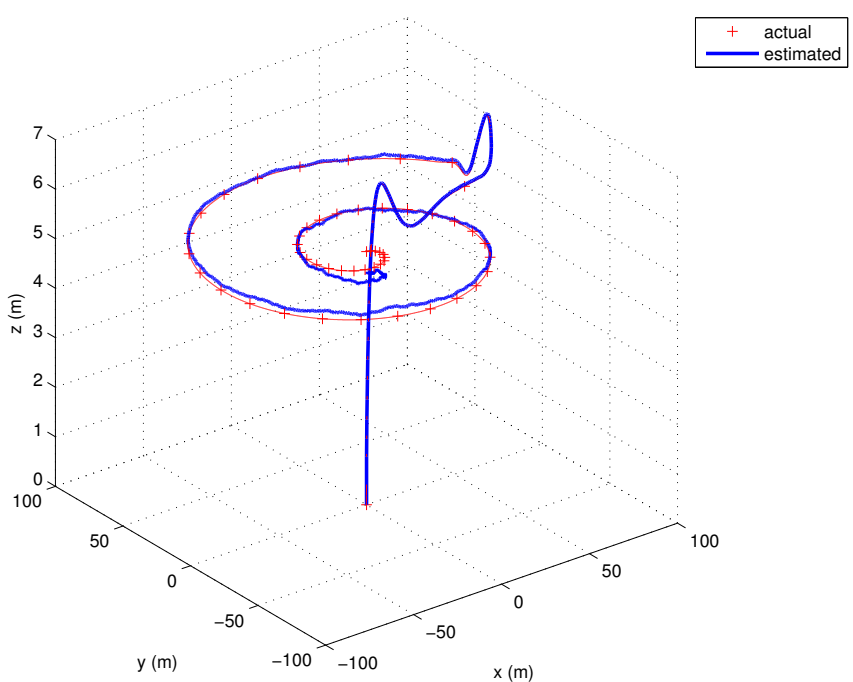

Fig. 8. 3d.

UAV navigation with GPS/INS/optical flow integration. International Conference on Electrical and Control Engineering, 2010.

[9] J. Wang, M. Garratt, A. Lambert, J. Jianguo, S. Han \& D. Sinclair. Integration of GPS/INS/vision sensors to navigate unmanned aerial vehicles. In The International Archives of the Photogrammetry, Remote Sensing and Spatial Information Sciences. Vol. XXXVII. Part B1. Beijing 2008.

[10]

[11] Romo-Morales, L., Sanchez, A., Parra-Vega, V., Garcia, O. \& Ruiz-Sanchez, F. Visual Control for Trajectory Tracking of Quadrotors and Real-Time Analysis on an Emulated Environment. American Control Conference (ACC), Washington, USA, 2013

F. Kendoul, D. Lara, I. Fantoni \& R. Lozano. Nonlinear control for systems with bounded inputs: real-time embedded control applied to UAVs. In Proceedings of the 45th IEEE Conference on Decision and Control, 2006.

[12] J. Roskam. Airplane Flight Dynamics and Automatic Flight Controls, Part I, Roskam Aviation and Engineering Corporation, ch. 2, pp 25-31. USA, 1982.

[13] Chen, Z. "Bayesian filtering: From kalman filters to particle filters, and beyond". Technical report. McMaster University, 2003. 47.

[14] Kitagawa, G. "Monte Carlo filter and smoother for nonGaussian nonlinear state space models". Journal of Computational and Graphical Statistics, vol. 5, no. 1, pp. 125, 1996. 